\title{
Effectiveness of a brief psychotherapeutic intervention compared with treatment as usual for adolescent nonsuicidal self-injury: a single-centre, randomised controlled trial
}

\author{
Michael Kaess ${ }^{1,2}$ D $\cdot$ Alexandra Edinger ${ }^{2} \cdot$ Gloria Fischer-Waldschmidt $^{3} \cdot$ Peter Parzer $^{3} \cdot$ Romuald Brunner $^{4}$. \\ Franz Resch $^{3}$
}

Received: 15 April 2019 / Accepted: 30 August 2019 / Published online: 11 September 2019

(c) The Author(s) 2019

\begin{abstract}
Although nonsuicidal self-injury (NSSI) is a clinically significant behavior, evidence-based, specific, time-, and cost-effective treatment approaches are lacking. The aim of this study was to compare the efficacies of a brief cognitive-behavioral psychotherapy manual, the Cutting Down Programme (CDP), and treatment as usual (TAU) in the treatment of adolescent NSSI. We conducted a single-centre randomised controlled trial (RCT). Eligible participants were aged 12-17 years engaging in repetitive NSSI (at least 5 times within the past 6 months). We randomly allocated 74 participants to CDP ( $n=37$ ) or TAU ( $n=37$; in a 1:1 ratio). Outcome measures were administered before treatment (T0), directly after CDP or 4 months after baseline evaluation in the TAU group (T1), and another 6 months later (T2; primary endpoint). Primary outcome was a 50\% reduction in NSSI frequency within the past 6 months at 10-month follow-up (T2). Regarding the primary outcome, there were no significant differences between the CDP $(n=26 ; 70.3 \%)$ and TAU group $\left[n=27 ; 73.0 \% ; \chi^{2}(1)=0.07 ; p=0.797\right]$; NSSI frequency within the past 6 months was significantly reduced at T2 $\left[\chi^{2}(1)=12.45 ; p<0.001\right]$ with no between-group difference $\left[\chi^{2}(1)=0.14 ; p=0.704\right]$. However, we found a significant group x point of measurement interaction $\left[\chi^{2}(2)=7.78\right.$; $p=0.021]$ regarding NSSI within the last month indicating at T1. CDP was equally effective and achieved faster recovery compared to a significantly more intensive TAU in treating adolescent NSSI. The CDP could provide a brief and pragmatic first treatment within a stepped-care model for NSSI in routine clinical care.

Clinical Trial Registration The trial was prospectively registered in the German Registry of Clinical Trials (https://www. drks.de; DRKS00003605) and is now complete.
\end{abstract}

Keywords Nonsuicidal self-injury $\cdot$ Randomised controlled trial $\cdot$ Adolescents $\cdot$ Psychotherapy

\section{Introduction}

Electronic supplementary material The online version of this article (https://doi.org/10.1007/s00787-019-01399-1) contains supplementary material, which is available to authorized users.

Michael Kaess

michael.kaess@upd.ch

1 University Hospital of Child and Adolescent Psychiatry and Psychotherapy, University of Bern, Stöckli, Bolligenstrasse 141c, 3000 Bern 60, Switzerland

2 Section for Translational Psychobiology in Child and Adolescent Psychiatry, Clinic of Child and Adolescent Psychiatry, Centre for Psychosocial Medicine, University Hospital Heidelberg, Heidelberg, Germany
Nonsuicidal self-injury (NSSI) is defined "as the deliberate, self-inflicted damage of body tissue without suicidal intent and for purposes not socially or culturally sanctioned" (International Society for the Study of Self-Injury, ISSS).

3 Clinic of Child and Adolescent Psychiatry, Centre for Psychosocial Medicine, University Hospital Heidelberg, Heidelberg, Germany

4 Clinic and Policlinic of Child and Adolescent Psychiatry, Psychosomatics and Psychotherapy, University of Regensburg, Regensburg District Hospital, Regensburg, Germany 
According to a large systematic review, approximately $17-18 \%$ of adolescents worldwide report at least a single episode of NSSI during lifetime [1]. NSSI is associated with a variety of psychiatric disorders and an elevated risk of suicidal behavior [2]

To date, there is no universally agreed best practice for the treatment of NSSI [3]. A recent systematic review revealed that there are effective treatments, which include or can be expanded to include the treatment of NSSI. However, these approaches were mostly neither developed for nor do they specifically focus on NSSI [4]. Particularly, in the context of (emerging) borderline personality disorder (BPD), NSSI is often treated with dialectical behavior therapy for adolescents (DBT-A), which was found to be effective within a randomised controlled trial (RCT) [5]. These treatment effects remained stable within an 1-year follow-up [6]. Other treatments that seem effective are mentalization-based treatment for adolescents (MBT-A) and cognitive-behavioral therapy (CBT) $[3,7]$.

The need for our trial is illustrated on the example of DBT-A: one of the first aims within the DBT-A target hierarchy is the elimination of severe NSSI. In further stages, however, DBT-A targets a broad spectrum of BPD symptoms beyond NSSI. Therefore, this approach is rather extensive. In addition, DBT-A requires intensive additional training for therapists. Hence, access to this treatment approach is restricted due to limited resources and a shortage of welltrained clinicians [8]. Considering the lack of such specialized treatments available as well as the transdiagnostic character of NSSI [9], less intensive and easy accessible treatments that address NSSI beyond BPD are essential. Such specific treatment approaches may improve the general standard of care, especially for adolescents who are often struggling to receive adequate professional help [10].

A randomised controlled pilot study investigated the effectiveness of a brief behavioral intervention for NSSI in young adults (Treatment for Self-injurious Behaviors, T-SIB) [11]. It was found that T-SIB was moderately effective for decreasing NSSI [11]. However, the study only enrolled a small number of participants, and did not focus on the critical period of adolescence [12].

In 1999, a cognitive-oriented and problem-focused shortterm psychotherapy was developed for adults exhibiting nonsuicidal as well as suicidal self-injury [manual-assisted cognitive-behavior therapy (MACT)] [13]. Thus, it is important to note that this approach did not focus specifically on NSSI, such as T-SIB, but in addition on suicidal self-injury (SSI). Within a first RCT $(n=34)$, the subjects in the MACT group $(n=18)$ had a significantly greater reduction in the frequencies of self-harm incidents, suicide attempts and depressive symptoms compared to the treatment as usual (TAU) group $(n=16)$ [13]. However, within another large RCT $(n=480)$, there were no significant differences to a TAU, so that the use of the MACT was not supported in the routine treatment of patients [14].

In 2011, the MACT was adapted for adolescents in "The Cutting Down Programme" (CDP). A pilot study investigated 25 adolescents aged $12-18$ years and provided preliminary evidence that the CDP may be efficacious in reducing NSSI, suicidality, comorbid depression, and trait anxiety [15]. Overall, 16 (64\%) participants completed the intervention with an average number of only 8.5 sessions in this study. Outcomes were assessed at baseline, at the end of the treatment and at a follow-up after 3 months. Another important result was that the intervention was highly acceptable to patients and therapists. However, the sample size was rather small and the study did not employ a randomized controlled design [15].

In the present study, we tested the efficacy of the CDP compared to a more intensive TAU within a RCT. Our primary hypothesis was that significantly more adolescents in the CDP group would exhibit clinically significant reductions (at least 50\%) in the frequency of NSSI within the last 6 months at follow-up (T2) than those in the TAU group. Primary outcome was a 50\% reduction in the frequency of NSSI within the last 6 months at T2.

\section{Methods}

\section{Study design}

The present monocentric RCT aimed to compare CDP with TAU of higher treatment intensity. Figure 1 illustrates the study design, and Fig. 2 contains the trial profile. The RCT was conducted at the outpatient clinic for Adolescent Risktaking and Self-harm behavior (AtR!Sk) at the Clinic of Child and Adolescent Psychiatry at the University Hospital Heidelberg, Germany. The authors assert that all procedures contributing to this work comply with the ethical standards of the relevant national and institutional committees on human experimentation and with the Declaration of Helsinki. All procedures involving human subjects/patients were approved by the institutional review board of the medical faculty at the University of Heidelberg (Ethics Committee

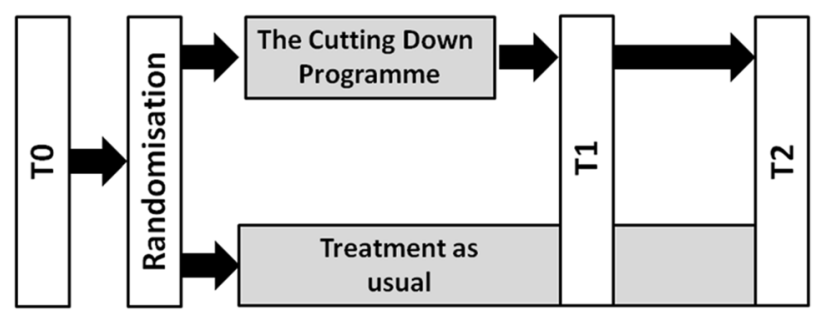

Fig. 1 Study design 


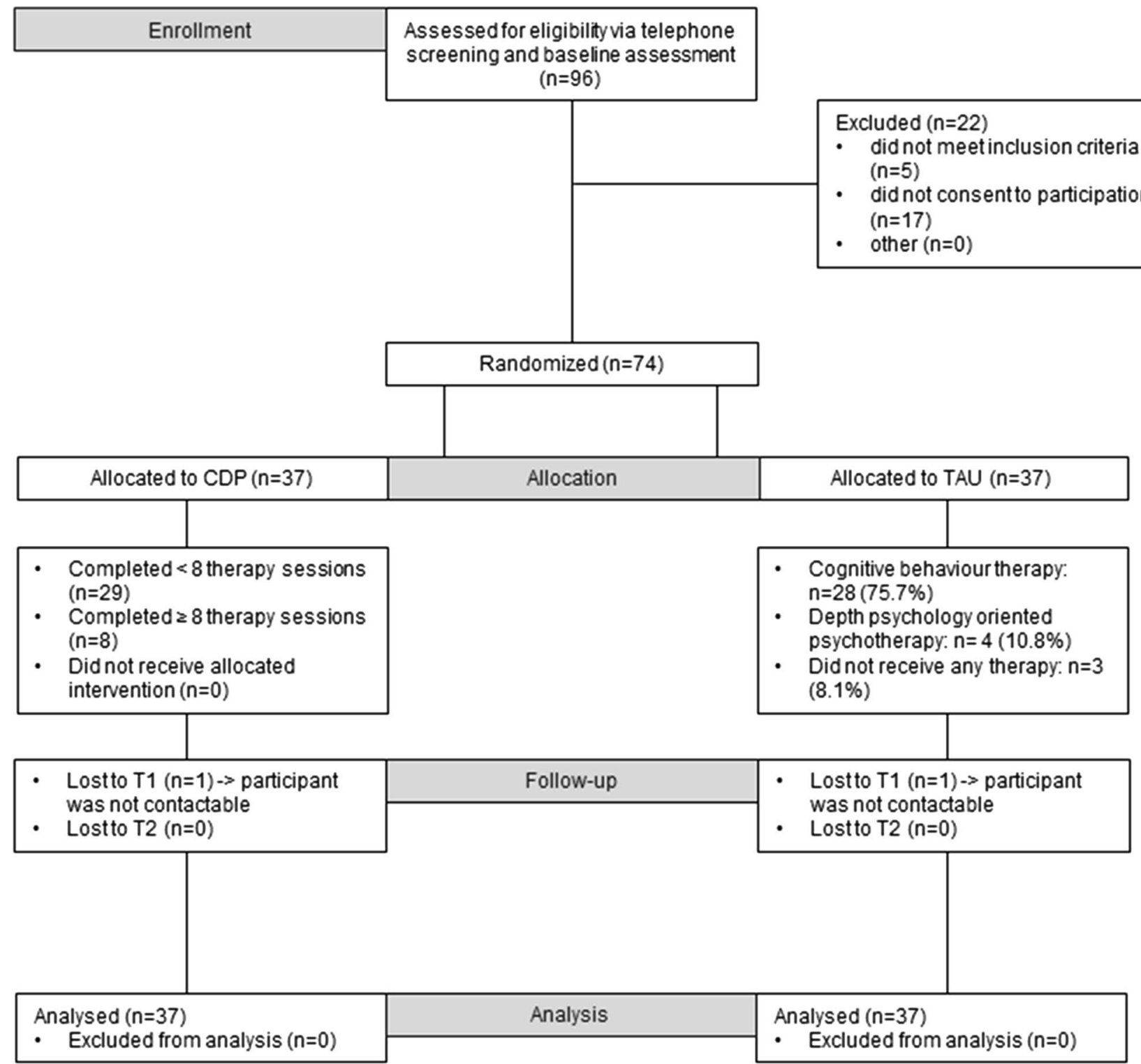

Fig. 2 Trial profile

No.: S-363/2011). The study design and procedures are presented in full in the published study protocol [16]. The trial was conducted and reported in accordance with CONSORT guidelines.

\section{Participant recruitment}

Participants were recruited via our in- and outpatient units, or using official notices, newspaper articles, advertisements, and study flyers. The eligible participants were between 12 and 17 years, and they were required to have engaged in NSSI at least 5 days during the previous 6 months and at least once during the past month. Exclusion criteria were acute psychotic symptoms, acute intent to harm self or others that requires intensive psychiatric inpatient treatment, impaired intellectual functioning, and/or currently receiving psychotherapeutic treatment. Eligibility was established by a clinical psychologist during a telephone screening, and if adolescents were suitable, written informed consent was appropriately obtained from all participants and caregivers (if participants were below 16 years of age) either via mail or during an information appointment. Subsequently, participants were invited to attend a baseline assessment.

\section{Assessments}

Participants were assessed at three timepoints: baseline (before randomisation, T0); after the completion of the 
CDP or 4 months after T0 within the TAU group to match the assessment points (T1); follow-up assessments (T2) 6 months after T1 (Fig. 2). To arrange for the T1 and T2 assessments, participants were contacted via phone. Participants received financial reimbursement following each assessment (T0-T2). All data were collected on paper forms and scanned for automatic data entry. Inconsistencies were resolved by checking the original paper material.

\section{Outcomes}

The primary outcome was a $50 \%$ reduction of NSSI frequency within the past 6 months at T2, via the German version of the Self-Injurious Thoughts and Behaviors Interview (SITBI-G) [17, 18]. This interview has been validated in adolescents (12-19 years) [17] and is considered reliable ( $\kappa s=0.77-1.00)$ [18]. The choice to use a $50 \%$ reduction in NSSI as an indicator of efficacy was made, because it is a clinical relevant outcome. This procedure is derived from the approach of Jacobson and Truax [19] which relates to supplementary information, namely, clinically relevant information over and above the information available from test statistics [20].

The secondary outcomes were a $50 \%$ reduction of NSSI within the past month, changes in NSSI frequency over time (within the last month and the last 6 months), suicide attempts within the last 6 months, depressive symptoms, and quality of life. NSSI and suicide attempts were assessed at every timepoint (T0-T2) using the SITBI-G. The German version of the Beck-Depression-Inventory-II (BDI-II) [21] was used to assess depressive symptoms at T0, T1, and T2. The BDI-II is considered reliable $(r=0.93)$ [21], and adequate for the use in adolescent samples [22]. The KIDSCREEN-27 questionnaire for children and adolescents (8-18 years of age) was used to assess subjective health and well-being at T0, T1, and T2. This version shows good internal consistency $(\alpha=0.70)$ [23].

Baseline assessment measures were comorbid mental disorders, which were assessed at T0 using the German version of the Mini-International Neuropsychiatric Interview for children and adolescents aged 6-17 years (M.I.N.I.-KID 6.0) $[24,25]$ and parts of the Structured Clinical Interview for DSM-IV-Axis II (SCID-II) [26]. In the present study, criteria for avoidant, dependent, borderline, and antisocial personality disorders were assessed. The M.I.N.I.-KID is a valid and reliable measure $(\kappa s=0.64-1.00)$ [24]. Although the SCID-II has been validated in adults [26], it is suitable for the use in adolescents [27] and reliable ( $\kappa s=0.77-0.94)$ [28].

Within AtR!Sk, assessors underwent specific training and regular reliability checks. To check for inter-rater reliability, audiotaped interviews of each clinician, consisting of the M.I.N.I.-Kid [24], the SCID II (borderline, avoidant, dependent, and antisocial personality disorder) [26], as well as the SITBI-G [17] were recorded. Interviews were assessed by independent second raters blind for the first raters' scores and diagnoses. Concerning the SITBI-G very good-to-perfect agreements were found within the interrater reliability checks ( $\kappa s=0.77-1.00)$ [18]. Regarding the SCID II, diagnostic agreement for full-threshold BPD was at $93.6 \%$ [29]. The clinician responsible for the interviews within the present study was involved in all inter-rater reliability checks mentioned in this section.

\section{Randomisation and masking}

Participants were randomised after completing the baseline assessment (T0). They were randomly assigned (in a 1:1 ratio) to receive either CDP or TAU using baseline adaptive randomisation. The probability of assignment to the CDP group was set to the proportion of designated CDP participants in the not yet randomized participants. This prevents large differences in group size during the time of the study and reduces the influence of seasonal effects. The randomisation was conducted by a researcher who was not involved in the diagnostic or treatment procedures using pseudorandom numbers generated by Stata 12 . The seed for the pseudorandom numbers was set to the time of day in seconds at the start of the randomisation. Allocation was undertaken using a password-protected website, independent of the trial. An independent staff member informed participants of the outcome of randomisation via telephone. All study assessments were performed by the same experienced and specially trained clinical psychologist at our clinic who was blinded to the participant's group allocation for the duration of the follow-up period using a separate and encrypted patient list. When asked after completion of interviews which treatment the assessor thought each patient received initially, the assessor's responses were correct for $49.1 \%$ of participants, indicating that masking was successful. Patients and clinicians were aware of treatment allocation.

\section{Treatment as usual}

The participants in the TAU group were referred to local cooperating child and adolescent psychotherapists and psychiatrists either in private practice or in psychotherapeutic institutes. All participating therapists and institutes committed themselves to providing the first appointment and subsequent psychotherapeutic treatments within 2-4 weeks after baseline assessment. Psychotherapeutic approaches included either CBT or psychodynamic oriented psychotherapy. Most of the participants received CBT ( $n=28,75.7 \%$; see Fig. 2). For detailed information on the TAU see Supplement 1. 


\section{Cutting Down Programme}

The manualized CDP that was applied in this study was based on elements of CBT and DBT and specifically tailored to the treatment of NSSI in adolescents. The treatment length was eight to 12 sessions. The manual organized the treatment into four modules that could be expanded with optional modules. Module 1 focused on promoting therapy motivation. The focus of module 2 was on identifying reasons for NSSI. In module 3, patients tested alternative behaviors to NSSI, and module 4 comprised stabilization of alternative behaviors [16]. The sessions were held once a week usually over a time of 2-4 months. Parental involvement was not specifically intended; however, appointments with parents were possible as needed. This decision was made by the therapist. Five therapists delivered 37 individual CDP therapies within our specialized outpatient clinic (AtR!Sk). For detailed information on the CDP see Supplement 2.

Study therapists were clinical psychologists (Master in clinical psychology) who were in training in CBT to become licensed psychotherapists. They already completed their intermediate exams after 1.5 years. In the context of the present study, they were trained in the CDP manual. Training consisted of two different aspects: in addition to the CDP treatment manual, there was a separate training manual to provide study therapists with detailed instructions and guidelines about the conduct of the treatment. Beyond that, an experienced clinical psychologist, who translated the manual, explicated the CDP training and treatment manual within a brief additional training session.

To measure adherence to the treatment manual, we used observational methods in terms of video recording and indirect measures. Video-based analyses of recorded therapy sessions were performed within supervision. An external supervisor, who was not involved in the study, checked the presented videos for adherence to the CDP manual. Types of rating were: occurrence of specific interventions (yes/ no) and frequency counts (how often an intervention occurs, expressed numerically). This was done once in a month. In addition, indirect adherence measures were used. Thus, the study therapists documented the specific exercises of each module as well as the corresponding work sheets in standardized psychotherapy notes, which were checked once a week by an independent staff member.

\section{Statistical analysis}

All analyses were intent-to-treat analyses (ITT). The study was powered to compare the effectiveness of the CDP and TAU in the treatment of NSSI. In secondary analyses, we used frequencies of NSSI in addition. We expected the CDP to result in greater reductions in NSSI incidents with a response rate difference of 35\% (e.g. CDP: $60 \%$; TAU:
$25 \%$ ). A response rate difference of 0.35 was considered to be clinically important. Similar rate differences were used within the power calculation of another RCT investigating CBT in patients with BPD [30]. To detect a rate difference of 0.35 with a power of $0.85(\alpha=0.05), 35$ participants were needed in each group.

Descriptive analyses were used to characterize the baseline study sample. Nominal data are presented as frequencies, while continuous data are presented as mean and standard deviation (SD) with confidence intervals. For variables with highly askew distribution, data are presented as medians and interquartile ranges. The influence of potential confounding variables was analyzed calculating regression models with and without covariates that were then compared using likelihood-ratio tests.

For our primary outcome, the TAU and CDP groups were compared with $\chi^{2}$ tests. Secondary outcomes were analyzed as follows. The changes in NSSI as well as in suicide attempts over time were analyzed with mixed-effects negative binomial regression because of the overdispersion of rates. In addition, to account for zero-inflation in suicide attempts, a zero-inflated negative binomial regression was calculated. To analyze a 50\% reduction in NSSI within the last month, both groups were compared with $\chi^{2}$ tests. Changes in depressive symptoms and quality of life over time were analyzed with mixed-effects multi-level regression. All analyses were performed with Stata (version 14; Stata Corp LLC, College Station, TX, USA).

Data were monitored by an independent researcher who was not involved in the study procedures. The trial was prospectively registered in the German Registry of Clinical Trials (https://www.drks.de; DRKS00003605) and is now complete.

\section{Role of the funding source}

The funder of the study had no role in study design, data collection, data analysis, data interpretation, or writing of the report. The corresponding author had full access to all the data in the study and had final responsibility for the decision to submit for publication.

\section{Results}

Between July 1, 2012, and February 1, 2016, of 96 participants assessed for eligibility, we recruited 74 participants. Of these, 37 participants were allocated to receive CDP and 37 participants were allocated to receive TAU (Fig. 2). Primary and secondary outcome data were obtained for 37 (100\%) participants in the CDP and 37 (100\%) participants in the TAU group. We retained 74 (100\%) of 74 participants over the 10-month follow-up period. 
Baseline characteristics were balanced between the two groups (Table 1).

Table 2 shows the number of completed therapy sessions as well as rates of supporting medication. The TAU group completed more therapeutic sessions on average than the CDP group did $(p=0.021)$, which indicates higher treatment intensity in the TAU group as expected. Concerning medication, there were no differences between the groups.
Analysis of the primary outcome showed that the majority of participants in both groups reached a $50 \%$ reduction in NSSI (TAU: $n=27 ; 73.0 \%$ vs. CDP: $n=26 ; 70.3 \%$ ). Contrary to our hypothesis, there was no difference between the two groups $\left[\chi^{2}(1)=0.07 ; p=0.797\right]$.

$50 \%$ NSSI reduction within the last month Within the CDP group, significantly more participants $(n=28 ; 75.0 \%)$ exhibited reductions of at least $50 \%$ in the frequency of NSSI within the last month at $\mathrm{T} 1\left[\chi^{2}(1)=4.25 ; p=0.039\right]$ than the
Table 1 Sociodemographic and clinical sample characteristics at T0

\begin{tabular}{|c|c|c|c|c|c|c|}
\hline \multirow{2}{*}{$\begin{array}{l}\text { Sociodemographic variable/diagnostic category } \\
\text { Age }\end{array}$} & \multicolumn{2}{|c|}{ TAU $(N=37)$} & \multicolumn{2}{|c|}{$\operatorname{CDP}(N=37)$} & \multicolumn{2}{|c|}{$\begin{array}{l}\text { Total } \\
(N=74)\end{array}$} \\
\hline & M & SD & M & SD & M & SD \\
\hline & 15.2 & 1.1 & 14.6 & 1.3 & 14.9 & 1.2 \\
\hline Sex & $N$ & $\%$ & $N$ & $\%$ & $N$ & $\%$ \\
\hline Female & 34 & 91.9 & 37 & 100.0 & 71 & 96.0 \\
\hline Male & 3 & 8.1 & 0 & 0.0 & 3 & 4.1 \\
\hline School type ${ }^{\mathrm{a}}$ & $N$ & $\%$ & $N$ & $\%$ & $N$ & $\%$ \\
\hline Hauptschule/foerderschule & 3 & 8.1 & 8 & 21.6 & 11 & 14.9 \\
\hline Realschule & 13 & 35.1 & 17 & 46.0 & 30 & 40.5 \\
\hline Gymnasium & 21 & 56.8 & 12 & 32.4 & 33 & 44.6 \\
\hline Migration status & $N$ & $\%$ & $N$ & $\%$ & $N$ & $\%$ \\
\hline Kazakhstan & 0 & 0.0 & 1 & 2.7 & 1 & 1.4 \\
\hline India & 0 & 0.0 & 1 & 2.7 & 1 & 1.4 \\
\hline Russia & 0 & 0.0 & 1 & 2.7 & 1 & 1.4 \\
\hline Spain & 0 & 0.0 & 1 & 2.7 & 1 & $1 \cdot 4$ \\
\hline Portugal & 1 & 2.7 & 0 & 0.0 & 1 & 1.4 \\
\hline Denmark & 1 & 2.7 & 0 & 0.0 & 1 & 1.4 \\
\hline Germany & 35 & 94.6 & 33 & 89.2 & 68 & 91.9 \\
\hline M.I.N.I.-Kid primary diagnoses ${ }^{\text {b }}$ & $N$ & $\%$ & $N$ & $\%$ & $N$ & $\%$ \\
\hline No diagnosis & 2 & 5.4 & 1 & 2.7 & 3 & 4.1 \\
\hline Current major depression & 16 & 43.2 & 11 & 29.7 & 27 & 36.5 \\
\hline Past major depression & 2 & 5.4 & 1 & 2.7 & 3 & 4.1 \\
\hline Recurrent depressive disorder & 2 & 5.4 & 6 & 16.2 & 8 & 10.8 \\
\hline Dysthymia & 9 & 24.3 & 7 & 18.9 & 16 & 21.6 \\
\hline Agoraphobia & 1 & 2.7 & 0 & 0.0 & 1 & 1.4 \\
\hline Social phobias & 1 & 2.7 & 1 & 2.7 & 2 & 2.7 \\
\hline Post-traumatic stress disorder & 1 & 2.7 & 1 & 2.7 & 2 & 2.7 \\
\hline Drug/alcohol dependence & 00.0 & & 12.7 & & 11.4 & \\
\hline ADHD & 1 & 2.7 & 0 & 0.0 & 1 & 1.4 \\
\hline Oppositional defiant disorder & 0 & 0.0 & 3 & 8.1 & 3 & 4.1 \\
\hline Affective disorders with psychotic features & 0 & 0.0 & 1 & 2.7 & 1 & 1.4 \\
\hline Bulimia nervosa & 0 & 0.0 & 1 & 2.7 & 1 & 1.4 \\
\hline Adjustment disorders & 2 & 5.4 & 3 & 8.1 & 5 & 6.8 \\
\hline \multicolumn{7}{|l|}{ SCID-II } \\
\hline Borderline personality disorder & 8 & 21.6 & 15 & 40.5 & 23 & 31.1 \\
\hline
\end{tabular}

$S D$ standard deviation

${ }^{a}$ Foerderschule: school for students with special needs; Hauptschule: nine years of elementary school; Realschule: six years of school after four years of elementary school, terminating with a secondary school level-I certificate; Gymnasium: eight years of school after four years of elementary school, terminating with the general qualification for university entrance

${ }^{\mathrm{b}}$ Multiple diagnoses per subject possible 
Table 2 Participation in intervention programs and effects on clinical outcomes

\begin{tabular}{|c|c|c|c|c|c|}
\hline Intervention/clinical outcome & TAU & & CDP & & Group differences \\
\hline Treatment adherence & M & SD & M & SD & $p$ value $^{\mathrm{a}}$ \\
\hline Mean number of sessions attended to T1 & 5.0 & 4.3 & 9.6 & 2.7 & $<0.001$ \\
\hline Mean number of sessions attended to $\mathrm{T} 2$ & 14.3 & 13.0 & 3.3 & 6.5 & $<0.001$ \\
\hline Total mean number of sessions attended & 19.3 & 14.0 & 12.9 & 7.9 & 0.021 \\
\hline Medication (subjects) & $N$ & & $N$ & & $P$ value \\
\hline $\mathrm{T} 1$ & & & & & 0.693 \\
\hline Antidepressants & 2 & & 1 & & \\
\hline Neuroleptics & - & & - & & \\
\hline Methylphenidate & - & & 1 & & \\
\hline $\mathrm{T} 2$ & & & & & 0.258 \\
\hline Antidepressants & 4 & & 4 & & \\
\hline Neuroleptics & - & & 1 & & \\
\hline Methylphenidate & - & & 2 & & \\
\hline NSSI in last 6 months & Median & IQR & Median & IQR & $\begin{array}{l}p \text { value } \\
0.461\end{array}$ \\
\hline T0 & 60 & $30-95$ & 50 & $25-90$ & \\
\hline $\mathrm{T} 2$ & 8 & $1-50$ & 10 & $2-40$ & \\
\hline NSSI in last month & Median & IQR & Median & $\begin{array}{l}\text { IQR } \\
0.565\end{array}$ & $p$ value \\
\hline T0 & 4 & $1-13$ & 10 & $4-15$ & \\
\hline $\mathrm{T} 1$ & 1 & $0-10$ & 1 & $0-3$ & \\
\hline $\mathrm{T} 2$ & 1 & $0-2$ & 0 & $0-2$ & \\
\hline Suicide attempts in last 6 months & M & SD & M & SD & $\begin{array}{l}p \text { value } \\
0.353\end{array}$ \\
\hline T0 & 0.4 & 0.8 & 0.5 & 0.8 & \\
\hline $\mathrm{T} 2$ & 0.3 & 0.7 & 0.1 & 0.4 & \\
\hline BDI-II scores & M & SD & M & SD & $\begin{array}{l}p \text { value } \\
0.980\end{array}$ \\
\hline T0 & 32.7 & 10.2 & 32.9 & 11.7 & \\
\hline $\mathrm{T} 1$ & 27.1 & 12.4 & 25.1 & 15.0 & \\
\hline $\mathrm{T} 2$ & 20.9 & 14.9 & 22.8 & 13.9 & \\
\hline KIDSCREEN-27 & M & SD & M & SD & $\begin{array}{l}p \text { value } \\
0.774\end{array}$ \\
\hline T0 & 38.1 & 5.4 & 39.0 & 7.0 & \\
\hline $\mathrm{T} 1$ & 41.0 & 6.2 & 42.6 & 7.5 & \\
\hline $\mathrm{T} 2$ & 44.7 & 8.4 & 43.7 & 8.9 & \\
\hline
\end{tabular}

$I Q R$ interquartile ranges

${ }^{\mathrm{a}}$ Group differences regarding primary and secondary outcome criteria participants in the TAU group did $(n=19 ; 51.4 \%)$. At T2, the difference between the groups was no longer significant $\left[\chi^{2}(1)=2.09 ; p=0.148\right]$.

NSSI frequencies within the last month and the last 6 months Medians and interquartile ranges are shown in Table 2. Although both groups exhibited significant reductions in the frequency of NSSI incidents within the previous 6 months $\left[\chi^{2}(1)=12.45 ; p<0.001\right]$, reductions did not differ significantly between the groups $\left[\chi^{2}(1)=0.14 ; p=0.704\right]$. Both groups also exhibited significant reductions in the NSSI frequencies within the last month $\left[\chi^{2}(2)=53.54\right.$; $p<0.001$; see Fig. 3]. In addition, we found a significant group $\times$ point of measurement interaction $\left[\chi^{2}(2)=7.78\right.$; $p=0.021]$ regarding NSSI incidents within the last month. Thus, the CDP group exhibited a faster reduction in the frequency of NSSI incidents compared with the TAU group. This group $\times$ point of measurement interaction remained stable when controlling for the number of therapy sessions $\left[\chi^{2}(2)=8.77 ; p=0.012\right]$.

Effect sizes for the reduction of NSSI frequency were large in both groups (TAU: Cohen's $d=0.79$; CDP: Cohen's $d=0.99$ ). 

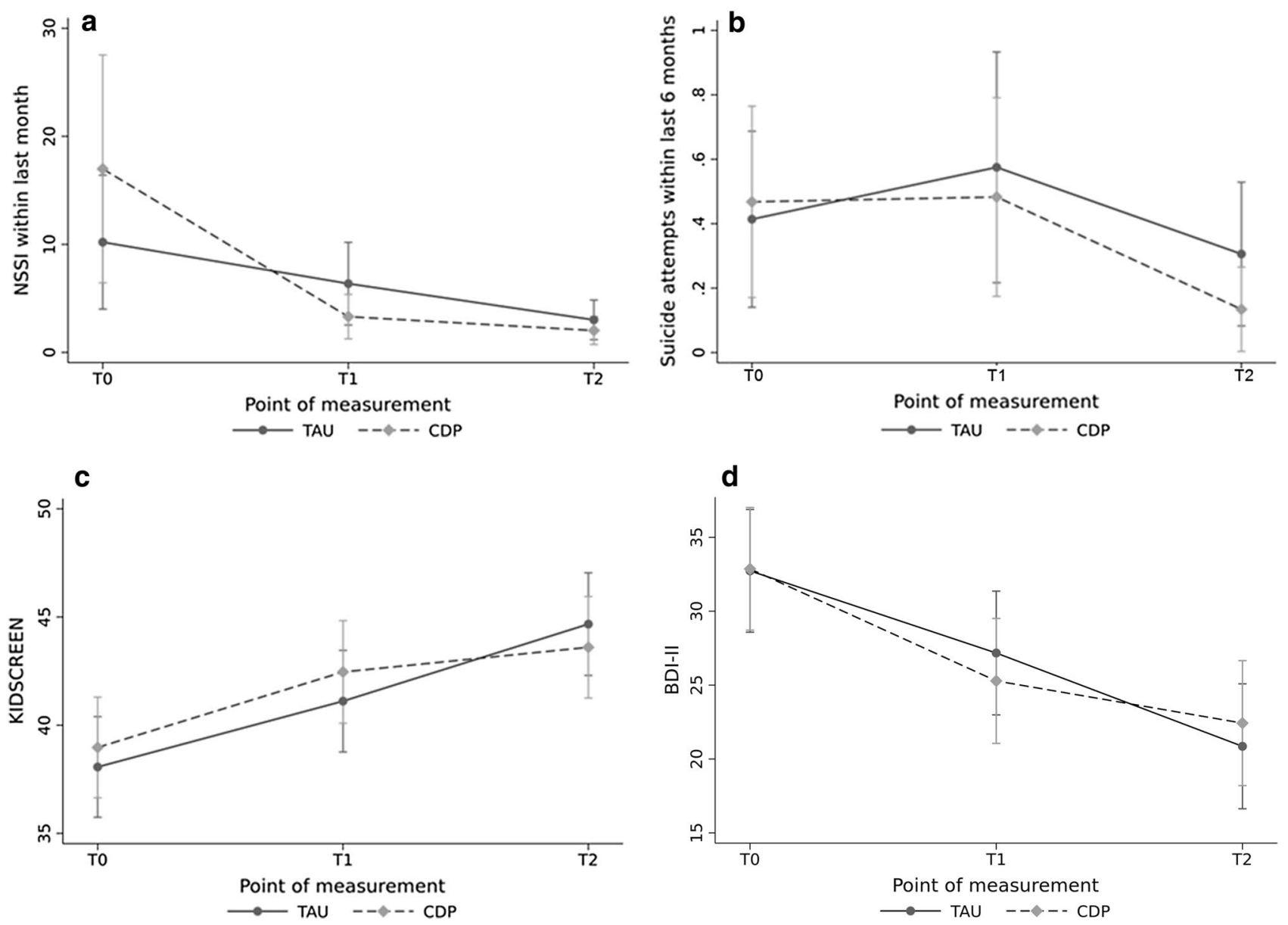

Fig. 3 Secondary outcomes: change of NSSI frequencies within the last month, suicide attempts, quality of life, and depression

Attempted suicides within the last 6 months As shown in Table 2 and Fig. 3, both groups demonstrated decreased attempted suicides within the last 6 months over time $\left[\chi^{2}(2)=6.76 ; p=0.034\right]$, with no significant difference between the treatment groups $\left[\chi^{2}(1)=0.86 ; p=0.353\right]$. This was also true when accounting for zero-inflation (Coef. $-0.57,95 \% \mathrm{CI}-1.43$ to $0.29, p=0.195$ ).

Depressive symptoms At T0, both groups were severely depressed (Table 2). As shown in Fig. 3, both the TAU and CDP groups reported significant reductions in depression over time [TAU: T0: 32.7; T1: 27.1; T2: 20.9; CDP: T0: 32.9; T1: $\left.25.1 ; \mathrm{T} 2: 22.8 ; \chi^{2}(2)=55.62 ; p<0.001\right]$ with again no group difference $\left[\chi^{2}(1)=0.00 ; p=0.980\right]$.

Quality of life The T0 assessment using the KIDSCREEN-27 showed that both groups had similar levels of well-being. As shown in Fig. 3 and Table 2, quality of life increased significantly for both groups over time $\left[\chi^{2}(2)=43.70 ; p<0.001\right]$, without any group differences $\left[\chi^{2}(1)=0.08 ; p=0.774\right]$.

There were no adverse events related to the interventions of the trial.
To control for potential confounding variables, we took treatment dose, BPD as well as depression as covariates within separate regression models into account, and compared them with the respective models without covariates. In general, models including covariates predicting the primary outcome did not differ from those without covariates (treatment dose: $p=0.851$; BPD: $p=0.095$; depression (BDI-II): $p=0.524)$. The same applied to the regression models that included the significant group $\times$ point of measurement interaction regarding NSSI incidents within the last month [treatment dose: $p=0.312$; BPD: $p=0.407$; depression (BDI-II): $p=0.142]$. For further information on analyses of confounding variables see Supplement 3.

\section{Discussion}

At T2, there was no evidence for the superiority of CDP compared with a significantly more intensive TAU for adolescents with repetitive NSSI. This was true for the primary outcome of a $50 \%$ reduction in NSSI frequency within the 
past 6 months as well as any of the secondary outcomes. Before this study, no study had compared the CDP with TAU.

Within a prior RCT with a 6-month follow-up, the adult version of the CDP, the MACT $(n=18)$, was compared to TAU $(n=16)$ and resulted in lower relapse rates regarding suicidal acts (56\% for MACT vs. $71 \%$ for TAU) [13]. Beyond that, the CDP was investigated within a pilot study among 25 adolescents with repetitive NSSI. Results suggested preliminary evidence that the CDP may reduce NSSI, depression and trait anxiety [15].

The present findings showed that outpatient treatment with either CDP or TAU significantly and equally decreased NSSI, suicide attempts, and depression symptoms as well as significantly increased quality of life with large effect sizes at $\mathrm{T} 2$. These results are consistent with data showing that psychotherapeutic interventions are effective for treating NSSI [3]. T1 assessments, however, showed that the CDP group exhibited faster reductions in the frequency of NSSI within the last month. Results in the CDP group remained stable at T2 suggesting that the effects on NSSI endure even after termination of a brief intervention.

In our study, CDP patients received a significantly lower treatment dose (i.e., psychotherapy sessions) compared to the TAU patients. However, there was no effect of treatment dose on outcomes indicating that successful treatment of NSSI can be ensured using a brief psychotherapeutic intervention. Comparing our trial with previous studies, patients treated with MBT-A and DBT-A during previous RCTs had also completed significantly more therapeutic sessions compared to CDP patients who only completed a mean of 10 sessions $[6,7]$. In addition, the TAU groups examined in other RCTs of NSSI provided a similar number of sessions compared to the respective index group $[5,7]$. Our and other TAU groups were clinical samples with comparable group sizes [5, 7]. Regarding outcomes, however, the effect sizes for the reduction in the frequency of NSSI in previous TAU groups were rather small in the other RCTs (Cohen's $d$ between 0.23 and 0.40 ) $[5,7]$ compared to the large effect sizes found in the present study (Cohen's $d=0.79$ ). Finally, trials on both MBT-A and DBT-A revealed no superiority regarding celerity of NSSI reduction compared to the TAU groups $[6,7]$.

This RCT has high ecological validity concerning the majority of patients engaging in NSSI. Since NSSI can occur in the context of various disorders [12] and is commonly associated with suicidality [2], we did not exclude patients based on comorbid disorders or suicidality (except acute symptoms that prohibited outpatient treatment). Secondary analyses indicated that treatment effects were independent of major comorbid disorders [e.g., BPD or major depressive disorder (MDD)]. Thus, the CDP seems effective for treating NSSI in the context of a broad spectrum of comorbid diagnoses. Furthermore, our study focused on mid-adolescence, during which NSSI prevalence rates peak, while help-seeking is commonly low [10]. The internal validity of the trials was established through the fidelity of the CDP delivery, high rates of treatment adherence, perfect retention, and through masked outcome assessment. The external validity was maximized by eliminating waiting times within the TAU group and a 10-month follow-up.

This study had several limitations. First, because the sample predominantly consisted of female participants, conclusions cannot be made on possible gender differences. However, considering that the female gender has been identified as a risk factor for NSSI, the present sample depicted this finding [12]. Furthermore, we were not able to control for the naturalistic course of NSSI, because psychotherapeutic interventions are adequate and effective in treating NSSI [3]. Thus, it would not have been ethically defensible to allow participants to wait 10 months before receiving treatment. NSSI increases in early adolescence and decreases in late adolescence with a peak in mid-adolescence (15-16 years) [12]. Because our study sample had a mean age of 14.9 years $(\mathrm{SD}=1.2)$ at $\mathrm{T} 0$, the frequency of NSSI might also have declined due to its natural course. In addition, the assumed rate difference between the CDP and TAU groups was rather optimistic. However, it should be noted that the group difference in response rate that we finally found in our trial was very small $(2.7 \%)$; thus, a far larger sample would not have led to statistically differences in the main outcome.

In a rigorously conducted $\mathrm{RCT}$, we have shown that CDP is not superior to TAU with higher treatment intensity over a 10-month follow-up for adolescent participants with repetitive NSSI but both treatments were associated with enduring positive outcomes in terms of a significant reduction in NSSI frequency, suicide attempts and depression as well as a significant improvement in quality of life. In addition, the CDP reached faster reductions in NSSI frequency compared to TAU. As a conclusion, the CDP showed similar treatment outcomes with a faster recovery while requiring less treatment sessions. This study provides important evidence that CDP might be an easily accessible, quickly available treatment for patients who would like an alternative to TAU. The brevity and potential cost-effectiveness of the CDP alongside its non-inferiority may nonetheless justify the implementation of such a brief psychotherapy manual in existing health care systems. As an example, the CDP could complement conventional interventions in terms of a stepped-care model to provide all concerned persons with easily accessible and quick professional help before performing more extensive treatments which can then be applied on those who really need them.

Acknowledgements The study was funded by the Dietmar Hopp Foundation (Grant number23011188) and sponsored by additional research 
funds of the Clinicfor Child and Adolescent Psychiatry at the University of Heidelberg. We thank Ulrike Schmidt and Lucy Taylor at the King's CollegeLondon for providing the English version of the "The Cutting-DownProgramme".

Author contributions MK was responsible for the study design and coordination. MK and AE wrote the first draft. AE and GFW participated in recruitment and assessment. PP participated in the design of the study and performed the statistical analyses. RB and FR participated in the design of the study and supervised the study procedure. All authors revised the article critically and approved the final version of the manuscript.

\section{Compliance with ethical standards}

Conflict of interest The authors declare that they have no conflict of interest.

Ethical standards Written informed consent was appropriately obtained from all participants and caregivers (if participants were below 16 years of age) prior to study inclusion. The study protocol has been approved by the institutional review board of the medical faculty at the University of (Ethics Committee No.: S-363/2011) and, therefore, has been performed in accordance with the ethical standards laid down in the 1964 Declaration of Helsinki and its later amendments.

Open Access This article is distributed under the terms of the Creative Commons Attribution 4.0 International License (http://creativeco mmons.org/licenses/by/4.0/), which permits unrestricted use, distribution, and reproduction in any medium, provided you give appropriate credit to the original author(s) and the source, provide a link to the Creative Commons license, and indicate if changes were made.

\section{References}

1. Swannell SV, Martin GE, Page A et al (2014) Prevalence of nonsuicidal self-injury in nonclinical samples: systematic review, meta-analysis and meta-regression. Suicide Life Threat Behav 44:273-303. https://doi.org/10.1111/sltb.12070

2. Koenig J, Brunner R, Fischer-Waldschmidt G et al (2017) Prospective risk for suicidal thoughts and behaviour in adolescents with onset, maintenance or cessation of direct self-injurious behaviour. Eur Child Adolesc Psychiatry 26:345-354. https:// doi.org/10.1007/s00787-016-0896-4

3. Ougrin D, Tranah T, Stahl D et al (2015) Therapeutic interventions for suicide attempts and self-harm in adolescents: systematic review and meta-analysis. J Am Acad Child Adolesc Psychiatry 54:97-107.e2. https://doi.org/10.1016/j.jaac.2014.10.009

4. Turner BJ, Austin SB, Chapman AL (2014) Treating nonsuicidal self-injury: a systematic review of psychological and pharmacological interventions. Can J Psychiatry 59:576-585. https://doi. org/10.1177/070674371405901103

5. Mehlum L, Tørmoen AJ, Ramberg M et al (2014) Dialectical behavior therapy for adolescents with repeated suicidal and self-harming behavior: a randomized trial. J Am Acad Child Adolesc Psychiatry 53:1082-1091. https://doi.org/10.1016/j. jaac.2014.07.003

6. Mehlum L, Ramberg M, Tørmoen AJ et al (2016) Dialectical behavior therapy compared with enhanced usual care for adolescents with repeated suicidal and self-harming behavior: outcomes over a one-year follow-up. J Am Acad Child Adolesc Psychiatry 55:295-300. https://doi.org/10.1016/j.jaac.2016.01.005

7. Rossouw TI, Fonagy P (2012) Mentalization-based treatment for self-harm in adolescents: a randomized controlled trial. J Am Acad Child Adolesc Psychiatry 51:1304-1313.e3. https://doi. org/10.1016/j.jaac.2012.09.018

8. Andreasson K, Krogh J, Wenneberg C et al (2016) Effectiveness of dialectical behavior therapy versus collaborative assessment and management of suicidality treatment for reduction of selfharm in adults with borderline personality traits and disordera randomized observer-blinded clinical trial. Depress Anxiety 33:520-530

9. Glenn CR, Klonsky ED (2013) Nonsuicidal self-injury disorder: an empirical investigation in adolescent psychiatric patients. J Clin Child Adolesc Psychol 42:496-507

10. Gulliver A, Griffiths KM, Christensen H (2010) Perceived barriers and facilitators to mental health help-seeking in young people: a systematic review. BMC Psychiatry. https://doi. org/10.1186/1471-244X-10-113

11. Andover MS, Schatten HT, Morris BW et al (2017) An intervention for nonsuicidal self-injury in young adults: a pilot randomized controlled trial. J Consult Clin Psychol. https://doi.org/10.1037/ ccp0000206

12. Plener PL, Schumacher TS, Munz LM, Groschwitz RC (2015) The longitudinal course of non-suicidal self-injury and deliberate self-harm: a systematic review of the literature. Borderline Pers Disord Emot Dysregulation 2:2. https://doi.org/10.1186/s4047 9-014-0024-3

13. Evans K, Tyrer P, Catalan J et al (1999) Manual-assisted cognitive-behaviour therapy (MACT): a randomized controlled trial of a brief intervention with bibliotherapy in the treatment of recurrent deliberate self-harm. Psychol Med 29:19-25

14. Tyrer P, Thompson S, Schmidt U et al (2003) Randomized controlled trial of brief cognitive behaviour therapy versus treatment as usual in recurrent deliberate self-harm: the POPMACT study. Psychol Med 33:969-976. https://doi.org/10.1017/S003329170 3008171

15. Taylor LM, Oldershaw A, Richards C et al (2011) Development and pilot evaluation of a manualized cognitive-behavioural treatment package for adolescent self-harm. Behav Cogn Psychother 39:619-625

16. Fischer G, Brunner R, Parzer P et al (2013) Short-term psychotherapeutic treatment in adolescents engaging in non-suicidal selfinjury: a randomized controlled trial. Trials 14:294. https://doi. org/10.1186/1745-6215-14-294

17. Nock MK, Holmberg EB, Photos VI, Michel BD (2007) Self-injurious thoughts and behaviors interview: development, reliability, and validity in an adolescent sample. Psychol Assess 19:309-317. https://doi.org/10.1037/1040-3590.19.3.309

18. Fischer G, Ameis N, Parzer P et al (2014) The German version of the self-injurious thoughts and behaviors interview (SITBI$\mathrm{G):} \mathrm{a} \mathrm{tool} \mathrm{to} \mathrm{assess} \mathrm{non-suicidal} \mathrm{self-injury} \mathrm{and} \mathrm{suicidal} \mathrm{behavior}$ disorder. BMC Psychiatry 14:265. https://doi.org/10.1186/s1288 8-014-0265-0

19. Jacobson NS, Truax P (1991) Clinical significance: a statistical approach to defining meaningful change in psychotherapy research. J Consult Clin Psychol 59:12

20. Hageman WJ, Arrindell WA (1999) Clinically significant and practical! Enhancing precision does make a difference. Reply to McGlinchey and Jacobson, Hsu, and Speer. Behav Res Ther 37:1219-1233

21. Beck AT, Steer RA, Brown GK (1996) Beck Depression Inventory - Second Edition. Manual. The Psychological Corporation, San Antonio: TX

22. Kumar G, Steer RA, Teitelman KB, Villacis L (2002) Effectiveness of Beck Depression Inventory-II subscales in screening for 
major depressive disorders in adolescent psychiatric inpatients. Assessment 9:164-170

23. Ravens-Sieberer U, European KG (2006) The KID-SCREEN questionnaires for children and adolescents - Handbook. Pabst Science Publishers, Lengerich

24. Sheehan DV, Sheehan KH, Shytle RD, et al (2010) Reliability and validity of the mini international neuropsychiatric interview for children and adolescents (MINI-KID). J Clin Psychiatry 71(3):313-326

25. Sheehan DV, Lecrubier Y, Sheehan KH et al (1998) The MiniInternational Neuropsychiatric Interview (M.I.N.I.): the development and validation of a structured diagnostic psychiatric interview for DSM-IV and ICD-10. J Clin Psychiatry 59(Suppl 20):22-33

26. Fydrich T, Renneberg B, Schmitz B, Wittchen H-U (1997) SKID II. Strukturiertes Klinisches Interview für DSM-IV, Achse II: Persönlichkeitsstörungen. Interviewheft. Eine deutschspeachige, erw. Bearb. d. amerikanischen Originalversion d. SKID-II von: M.B. First, R.L. Spitzer, M. Gibbon, J.B.W. Williams, L. Benjamin, (Version 3/96). Hogrefe, Göttingen
27. Chanen AM, Jackson HJ, McGorry PD et al (2004) Two-year stability of personality disorder in older adolescent outpatients. J Pers Disord 18:526-541

28. Lobbestael J, Leurgans M, Arntz A (2011) Inter-rater reliability of the structured clinical interview for DSM-IV axis I disorders (SCID I) and axis II disorders (SCID II). Clin Psychol Psychother 18:75-79

29. Kaess M, Fischer-Waldschmidt G, Resch F, Koenig J (2017) Health related quality of life and psychopathological distress in risk taking and self-harming adolescents with full-syndrome, subthreshold and without borderline personality disorder: rethinking the clinical cut-off? Borderline Pers Disord Emot Dysregulation $4: 7$

30. Davidson K, Tyrer P, Gumley A et al (2006) A randomized controlled trial of cognitive behavior therapy for borderline personality disorder: rationale for trial, method, and description of sample. J Pers Disord 20:431-449. https://doi.org/10.1521/ pedi.2006.20.5.431 